\title{
Skew Detection in Handwritten Documents
}

\author{
B. Aditya Vighnesh \\ Department of Electronics and \\ Communication Engineering \\ NIT Warangal \\ Warangal, AP, India
}

\author{
Abhishek Kumar \\ Department of Electronics and \\ Communication Engineering \\ NIT Warangal \\ Warangal, AP, India
}

\author{
B. Manikanta Yadav \\ Department of Electronics and \\ Communication Engineering \\ NIT Warangal \\ Warangal, AP, India
}

\begin{abstract}
In this paper, we describe a simple novel algorithm to detect the skew in hand-written documents.

\section{General Terms}

Document Analysis, Handwriting Analysis, Computer Vision

\section{Keywords}

Skew Detection, Handwritten Document Analysis, OCR, Handwriting Recognition (HWR).

\section{INTRODUCTION}

When a document is scanned, skew is inevitably introduced into the image due to various factors. Most of the popular algorithms for character recognition and layout analysis, however, are very sensitive to the distortion of document images and skew may cause serious problems for document analysis. Furthermore, it is very difficult, if not totally impossible, to prevent skew distortion produced in scanning procedures, it is therefore preferable to detect and correct the skew document image at the pre-processing stage in order to avoid the disturbance of skew to the further processing.
\end{abstract}

Several attempts have been made for skew detection, among which the methods based on Hough transformation are extremely popular. Hinds et al. [1] uses run-length coding and Hough transformation while Cao [2] uses line-fitting followed by Hough transformation for detecting skew.Other methods for skew detection include cross correlation used by Chen et al. [3], projection profile by Steinherz [4], Fourier transformation by W. Postl[5]. All of these methods give high accuracy but none of them focus on the memory constraints involved while processing the documents.

This paper presents a technique involving connected component analysis and vertical edges to detect skew where the accuracy is tunable depending on the memory constraints. This algorithm is on the same lines as the work done by Dhandra et al [6] as it is based on image dilation but is differs in it approach. The algorithm may be used in embedded biometric systems to identify characters or writers.

\section{SKEW DETECTION ALGORITHM}

The alignment of a document which may occur when scanned/photographed at an angle is known as skew.

\subsection{Our Algorithm}

- Convert the 300dpi scanned image into a binary image using necessary thresholds. The negative of the image is then formed. (Figure 1)

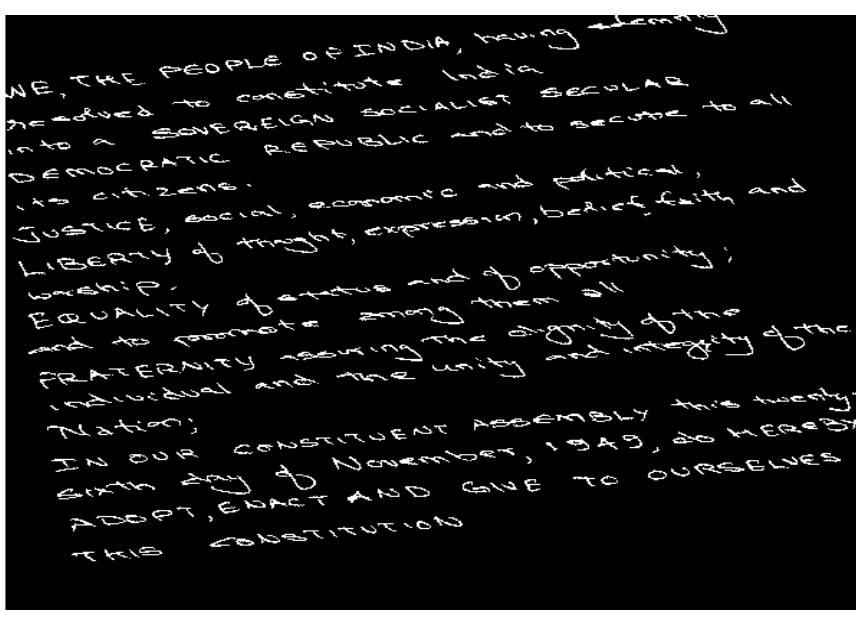

Figure 1: Skewed Handwritten Document

- The negative binary image is then dilated 10 times to form blobs (connected components). The resulting image is shown in Figure 2.

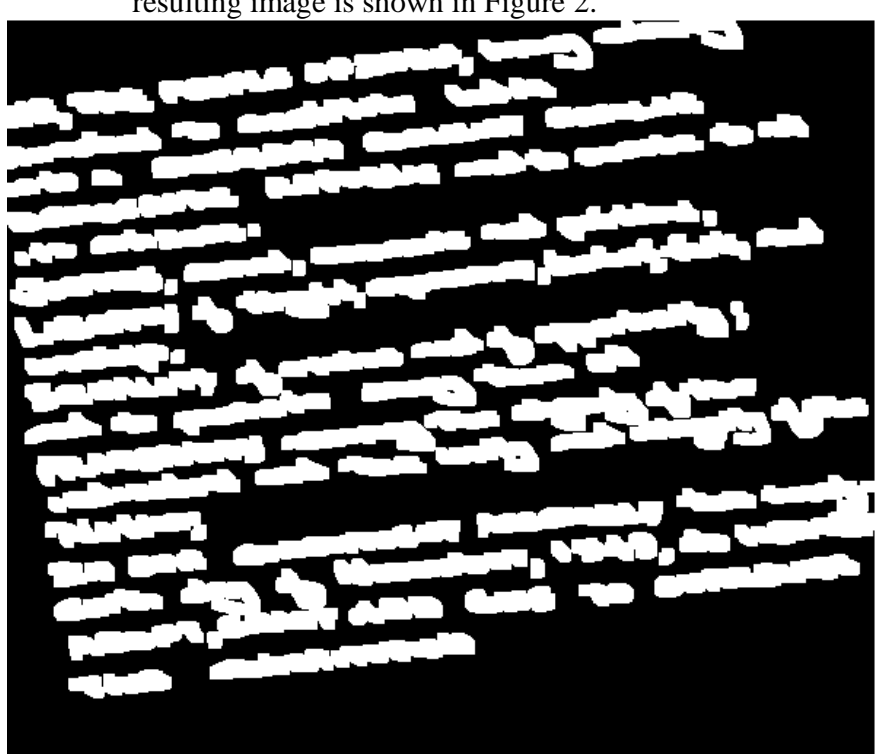

Figure 2: Formation of Connected Components 
- Find the major axis (longest line that could be drawn through the blob) of each blob.

- Calculate the orientation of the major axis with respect to the horizontal.

- Create bins for the different orientation angles to find the number of blobs having particular orientation.

- Find the bins having one less than the maximum number of blobs.

- The average of these bins gives the skew angle. In order to, remove the skew rotate the image in the opposite direction of the determined skew angle. (Figure 3 )

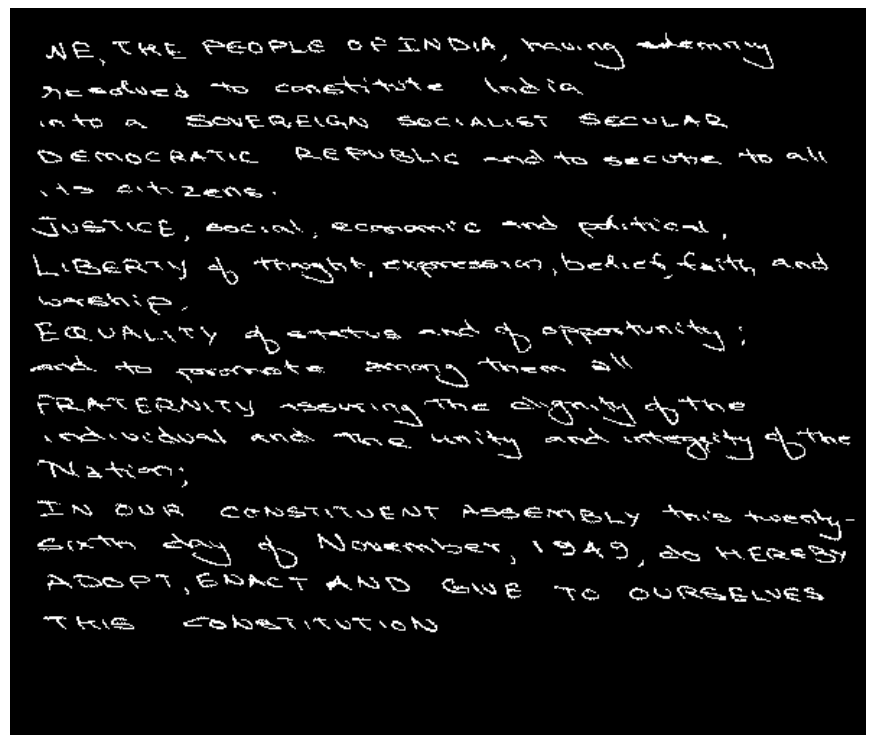

Figure 3: De-skewed Handwritten Document

\section{RESULTS}

The following results were obtained by simulating the algorithms in MATLAB 2010a on a laptop with an Intel Core i7 U640 @1.2 Ghz.

Table 1. Comparison of the algorithm accuracy

\begin{tabular}{|c|c|c|}
\hline $\begin{array}{c}\text { Skew Angle } \\
\text { (Degree) }\end{array}$ & $\begin{array}{c}\text { Hough } \\
\text { Transformation } \\
\text { Method }\end{array}$ & Our Method \\
\hline-5 & -5.1 & -5.5 \\
\hline-4 & -4 & -3.5 \\
\hline-0.1 & 0.0 & 0.0 \\
\hline 0.1 & 0.0 & 0.0 \\
\hline 5 & 4.9 & 5.5 \\
\hline
\end{tabular}

Table 2. Timing comparison of the algorithms

\begin{tabular}{|c|c|}
\hline Method & $\begin{array}{c}\text { Time to perform skew } \\
\text { correction of 3 degrees }\end{array}$ \\
\hline $\begin{array}{c}\text { Hough Transformation } \\
\text { Method }\end{array}$ & $10 \mathrm{~s}$ \\
\hline New Method & $3 \mathrm{~s}$ \\
\hline
\end{tabular}

\section{CONCLUSIONS}

Our skew detection algorithm is faster than Hough transformation method [1] [2] in terms of time taken. When compared to Dhandra's [6] approach our method is based on the orientation of each word rather than on the complete line. Moreover, in our algorithm the precision is tunable, i.e. it depends on the number of angle bins mentioned in the algorithm. This kind of tunability allows this algorithm to function on an embedded system for hand-writer recognition where the memory is constrained. Also this algorithm is robust for handwritten documents with two columns and graphics. The shortcomings of this algorithm are that it cannot detect a document skewed at 180 degrees. In future this issue may be addressed.

\section{ACKNOWLEDGMENTS}

We would like to acknowledge our Mentor, Sri M. V. Raghunadh, Associate Professor, Dept. of Electronics and Communication Engineering., NIT Warangal, without whose help and constant guidance this paper would not have been successfully completed.

\section{REFERENCES}

[1] Hinds, Stuart C., James L. Fisher, and Donald P. D'Amato. "A document skew detection method using run-length encoding and the Hough transform." Pattern Recognition, 1990. Proceedings, 10th International Conference on. Vol. 1. IEEE, 1990.

[2] Cao, Yang, Shuhua Wang, and Heng Li. "Skew detection and correction in document images based on straight-line fitting." Pattern Recognition Letters24.12 (2003): 18711879.

[3] Chen, Ming, and Xiaoqing Ding. "A robust skew detection algorithm for grayscale document image." Document Analysis and Recognition, 1999. ICDAR'99. Proceedings of the Fifth International Conference on. IEEE, 1999.

[4] Steinherz, Tal, Nathan Intrator, and Ehud Rivlin. "Skew detection via principal components analysis." Document Analysis and Recognition, 1999. ICDAR'99. Proceedings of the Fifth International Conference on. IEEE, 1999.

[5] W. Postl, "Detection of Linear Oblique Structure and Skew Scan in Digitized Documents". In Proc. 8th Int'l Conf. on Pattern Recognition, Pairs, France, Oct.27-31, 1986: pp687-689.

[6] Dhandra, B. V., et al. "Skew detection in Binary image documents based on Image Dilation and Region labeling Approach." Pattern Recognition, 2006. ICPR 2006. 18th International Conference on. Vol. 2. IEEE, 2006 